\title{
CONTRAVARIANTLY FINITE SUBCATEGORIES AND IRREDUCIBLE MAPS
}

\author{
JON F. CARLSON AND DIETER HAPPEL
}

(Communicated by Maurice Auslander)

\begin{abstract}
Let $\Lambda$ be an Artin algebra. This paper presents a condition that is necessary for a subcategory of $\bmod \Lambda$ to be contravariantly finite. Several examples are explored in the case that $\Lambda$ is the group algebra of a finite group over a field of finite characteristic.
\end{abstract}

\section{INTRODUCTION}

Several years ago Auslander and Smalø [4] introduced the notion of a contravariantly finite subcategory of a category. While the idea has had several applications $[3,5]$, it has occasionally been. difficult to decide when a subcategory satisfies the conditions necessary to make it contravariantly finite. At a recent meeting in Tsukuba this question was raised about some subcategories of the category of modules over a group algebra. From various methods of group representation theory it was shown that they were not contravariantly finite [6]. However, the proofs were indirect and provided no insight into the more general problem.

The main purpose of this paper is to provide a condition that is necessary for a subcategory to be contravariantly finite. The condition is given in terms of the left almost split morphism of the minimal approximation of an object not in the subcategory. Among other things it implies that subcategories that are closed under almost split morphisms are seldom contravariantly finite. In addition to the main theorem and its corollaries, we give a few examples that may be of independent interest. See [2] as a reference on almost split morphisms.

The second author would like to thank the University of Georgia for its hospitality during the visit in which the results of this paper were discovered.

\section{THE MAIN RESULT}

For convenience we shall work within the category $\bmod \Lambda$ of finitely generated modules over an Artin algebra $\Lambda$. However, the techniques will work just as well in any category that has almost split morphisms. By a subcategory we always mean a full subcategory that is closed under isomorphisms,

Received by the editors December 10, 1990 and, in revised form, May 28, 1991.

1991 Mathematics Subject Classification. Primary 16A64, 20C20.

The first author was partially supported by a grant from NSF. 
direct sums, and summands. Recall that a subcategory $\mathscr{C}$ of $\bmod \Lambda$ is contravariantly finite if every indecomposable object $M$ in $\bmod \Lambda$ has a right $\mathscr{C}$-approximation. By this we mean an object $U \in \mathscr{C}$ and a homomorphism $f: U \rightarrow M$ such that if $g: V \rightarrow M$ is any homomorphism with $V \in \mathscr{C}$ then there exists $\sigma: V \rightarrow U$ with $f \sigma=g$. It is known that there is always a minimal approximation that is a direct summand of any approximation.

The idea of a covariantly finite subcategory is dual to that of a contravariantly finite subcategory. The reader should recognize that all results of this paper have dual analogues for covariantly finite subcategories and left approximations.

Theorem 2.1. Suppose that $\mathscr{C}$ is a contravariantly finite subcategory of $\bmod \Lambda$. Let $M$ be any indecomposable module that is not in $\mathscr{C}$. Suppose that $f: X \rightarrow$ $M$ is the minimal $\mathscr{C}$-approximation and that $f \neq 0$. Then there exists an indecomposable summand $U$ of $X$ such that there is an irreducible map $g: U \rightarrow$ $V$ where $V$ is not in $\mathscr{C}$.

Proof. Let $\sigma: X \rightarrow Y$ be the left almost split morphism in $\bmod \Lambda$. Suppose that $Y$ is in $\mathscr{C}$. Our aim is to produce a contradiction.

With the above assumptions we have the following commutative diagram of $\Lambda$-modules and homomorphisms:

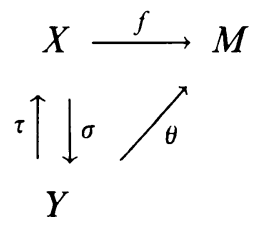

The existence of $\theta$ with $\theta \sigma=f$ is assured by the facts that $\sigma$ is left almost split and $f$ is not a splittable injection since $M$ is not in $\mathscr{C}$. Then the map $\tau$ with $f \tau=\theta$ exists because $Y$ is in $\mathscr{C}$ and $f: X \rightarrow Y$ is a right $\mathscr{C}$-approximation. Let $\mu=\tau \sigma$ and note that $f \mu=f$. The contradiction can now be derived from the fact that $f$ is the minimal approximation. A sketch of the details is as follows.

By the usual chain conditions there exists $n>0$ such that $\mu^{n}(X)=\mu^{n+j}(X)$ and $\operatorname{ker} \mu^{n}=\operatorname{ker} \mu^{n+j}$ for all $j \geq 0$. Clearly $\operatorname{ker} \mu^{n} \cap \mu^{n}(X)=\{0\}$ because otherwise $\mu^{2 n}(X)$ would have shorter composition length than $\mu^{n}(X)$. So $X=$ $X_{1} \oplus X_{2}$ where $X_{1}=\mu^{n}(X), X_{2}=\operatorname{ker} \mu^{n}$. In particular, $X_{1}$ is in $\mathscr{C}$ and, because the approximation factors through $X_{1}$, we have $X_{1}=X$ by minimality. But then $\mu^{n}: X \rightarrow X$ is an isomorphism and the left almost split map $\sigma: X \rightarrow$ $Y$ is split by $\left(\mu^{n}\right)^{-1} \circ \mu^{n-1} \circ \tau$. This is not possible and the contradiction proves the theorem.

Corollary 2.2. Suppose that a subcategory $\mathscr{C}$ is contravariantly finite and has the property that if $g: X \rightarrow Y$ is an irreducible map and if $X$ is in $\mathscr{C}$ then $Y$ is in $\mathscr{C}$. Then $\operatorname{Hom}_{\Lambda}(C, D)=\{0\}$ whenever $C$ is in $\mathscr{C}$ and $D$ is not in $\mathscr{C}$.

The proof of the corollary is obvious from the theorem. Recall that a regular component of the Auslander-Reiten graph is one that has no projective or injective objects.

Corollary 2.3. Let $\mathscr{C}$ be a subcategory whose collection of indecomposable objects is a union of regular Auslander-Reiten components. Then $\mathscr{C}$ is not contravariantly finite. 
The point of the last corollary is that for every object in $\mathscr{C}$ there is a nonzero morphism to an injective object that is not in $\mathscr{C}$. Hence the statement follows from the previous result.

We end this section by observing that our results give a new proof of the following well-known theorem.

Corollary 2.4 [1]. Suppose that the ring $\Lambda$ is connected and has a finite AuslanderReiten component. Then $\Lambda$ has finite representation type.

Proof. Let $\mathscr{C}$ be the subcategory generated by the finite component; that is, the objects of $\mathscr{C}$ are finitely generated summands of direct sums of objects in the component. Because $\mathscr{C}$ has only a finite number of indecomposable objects, $\mathscr{C}$ is contravariantly finite [3]. But then, by Corollary 2.2 and the connectedness of $\Lambda, \mathscr{C}=\bmod \Lambda$.

\section{CONSTRUCTING CONTRAVARIANTLY FINITE SUBCATEGORIES} AND SOME EXAMPLES

The results of the previous section point to a method for constructing contravariantly finite subcategories. The point is that we need only make certain that each Auslander-Reiten component that has modules that are not in the category also has enough modules that are in the category.

Proposition 3.1. Let $\mathscr{C}$ be a subcategory of $\bmod \Lambda$. Suppose that for any indecomposable module $M$ in $\bmod \Lambda$ but not in $\mathscr{C}$ there exists a positive integer $n$ such that if

$$
Y_{n} \rightarrow \cdots \rightarrow Y_{1} \rightarrow Y_{0} \rightarrow M
$$

is a chain of irreducible maps, then $Y_{i}$ is in $\mathscr{C}$ for some $0 \leq i \leq n$. Then $\mathscr{C}$ is contravariantly finite.

Proof. If $M$ is in $\mathscr{C}$ then Id: $M \rightarrow M$ is a right $\mathscr{C}$-approximation. So suppose that $M$ is indecomposable and $M$ is not in $\mathscr{C}$. Take a complete set $\left\{\left(X_{i}, f_{i}\right)\right\}_{i=1, \ldots, n}$ of representatives of the isomorphism classes of all possible pairs in which $X_{i}$ is in $\mathscr{C}$ and $f_{i}: X_{i} \rightarrow M$ is a composition

$$
X_{i}=Y_{n_{i}} \rightarrow \cdots \rightarrow Y_{0} \rightarrow M
$$

of irreducible maps such that $Y_{j}$ is indecomposable and not in $\mathscr{C}$ for $0 \leq j<$ $n_{i}$. Let $X=\bigsqcup_{i=1}^{n} X_{i}$ and define $f: X \rightarrow M$ by $\left.f\right|_{X_{i}}=f_{i}$. We claim that $f$ is a right $\mathscr{C}$-approximation for $M$. A sketch of the proof goes as follows.

Suppose that $Y$ is in $\mathscr{C}$ and that $g: Y \rightarrow M$ is a homomorphism. Let $\phi: \amalg B_{j} \rightarrow M$ be the right almost split morphisms so that $\left.\phi\right|_{B_{j}}=\phi_{j}$ is irreducible for all $j$. Because $g$ is not a splittable epimorphism there exists $\theta: Y \rightarrow \amalg B_{j}$ so that $\phi \theta=g$. Let $\theta_{j}$ be the composition $\theta$ with the projection onto $B_{j}$. So $\phi \theta=\sum \phi_{j} \theta_{j}=g$. If for a specific $j, B_{j}$ is in $\mathscr{C}$, then $\left(B_{j}, \phi_{j}\right) \cong\left(X_{i}, f_{i}\right)$ for some $i$. Hence $\phi_{j} \theta_{j}$ factors through $f$ in this case. Hence we need only worry about the case in which $B_{j}$ is not in $\mathscr{C}$. But now we can repeat the process. That is, let $\psi: \amalg C_{l} \rightarrow^{\psi} B_{j}$ be the right almost split morphism. Write $\theta_{j}=\sum\left(\left.\psi\right|_{C_{l}}\right) \circ \gamma_{l}, \gamma_{l}: Y \rightarrow C_{l}$. If $C_{l}$ is in $\mathscr{C}$ then $\left(C_{l},\left.\phi_{j} \circ \psi\right|_{C_{l}}\right) \cong\left(X_{i}, f_{i}\right)$ for some $i$. If $C_{l}$ is not in $\mathscr{C}$ then repeat the process again. The condition on the category $\mathscr{C}$ says that this must stop after a finite number of steps. 
Therefore to construct a contravariantly finite subcategory we need only take a full subcategory whose indecomposable objects are what remains after discarding a portion of some of the Auslander-Reiten components. We need only be certain that the condition of Proposition 3.1 is satisfied in each component. Hence, in the usual notation, we could discard the right "half" and the remaining modules would generate a contravariantly finite subcategory. We note, however, that not all such subcategories are constructed in this way. This can be seen from the examples given below.

Examples. In each of the following let $G$ be a finite group and let $K$ be a field of characteristic $p>0$. We assume that $p$ divides the order of $G$. Let $M$ be a $K G$-module. The module $\Omega(M)$ is defined to be the kernel of a surjection $P \rightarrow M$ where $P$ is a projective cover of $M$. Dually $\Omega^{-1}(M)$ is the cokernel of an injection $M \rightarrow Q$ where $Q$ is an injective hull of $M$ (which is also projective). We recall that the translation functor $\operatorname{Tr} D$ coincides with $\Omega^{-2}$. So if $M$ is indecomposable and not projective and if $\phi: M \rightarrow B$ is the left almost split morphism, then $B / \phi(M) \cong \Omega^{-2}(M)$.

Example 3.2. Let $\mathscr{C}$ be the full subcategory of $\bmod K G$ that is generated by all relatively $\mathscr{X}$-projective modules where $\mathscr{X}$ is some set of subgroups of $G$. Assume that no element of $\mathscr{X}$ contains a Sylow $p$-subgroup of $G$. It has long been known that $\mathscr{C}$ is contravariantly finite [9], however, it is not closed under irreducible maps. This last fact can be proved directly; but indirectly, it is a consequence of Corollary 2.3. That is, we notice that $\mathscr{C}$ is closed under translation by $\Omega$ and $\Omega^{-1}$. Hence, if it were also closed under irreducible maps then its collection of objects would be a union of Auslander-Reiten components. Observe that $\mathscr{C}$ also fails to satisfy the condition of Proposition 3.1. (See also [8].)

Example 3.3. Let $Q$ be a $p$-subgroup of $G$. Let $\mathscr{C}$ be the full subcategory of $\bmod K G$ generated by the modules whose restriction to $Q$ are free as $K Q$ modules. This subcategory is closed under extensions and also under translation by $\Omega$ and $\Omega^{-1}$. Hence its collection of objects, if we ignore projectives is a union of Auslander-Reiten components. Therefore, it is not contravariantly finite except in the extreme cases in which either $\mathscr{C}$ is the category of projective modules or $\mathscr{C}=\bmod K G$ (cf. [6]).

Example 3.4. Let $V$ be an indecomposable nonprojective $K G$-module. Let $\mathscr{C}$ be the full subcategory of $\bmod K G$ generated by the projective $K G$-modules and the modules $\Omega^{j}(V)$ for $j \geq 0$. Then $\mathscr{C}$ is contravariantly finite. A proof of this fact can be obtained as follows. The module $\operatorname{Ext}_{K G}^{*}(V, M)$ is finitely generated over the ring $\operatorname{Ext}_{K G}^{*}(V, V)$ [7]. Let $\alpha_{1}, \ldots, \alpha_{n}$ be a homogeneous set of generators. Then each $\alpha_{i}$ is represented by a cocycle $f_{i}: \Omega^{n_{i}}(V) \rightarrow M$ where $n_{i}=\operatorname{deg}\left(\alpha_{i}\right) \geq 0$. Let $X_{i}=\Omega^{n_{i}}(M)$ and set $X=\left(\coprod X_{i}\right) \oplus P_{M}$ where $P_{M}$ is a projective cover of $M$. Define $f: X \rightarrow M$ by $\left.f\right|_{X_{i}}=f_{i}$ and $\left.f\right|_{P_{M}}$ an epimorphism. We claim that this is a right $\mathscr{C}$-approximation of $M$.

Suppose that we are given a homomorphism $\theta: \Omega^{m}(V) \rightarrow M, m \geq 0$. Then $\theta$ represents a cohomology class $\hat{\theta}=\sum \alpha_{i} \gamma_{i}$ for some $\gamma_{i} \in \operatorname{Ext}_{K G}^{m-n_{i}}(V, V)$. Note here that $\gamma_{i}=0$ if $n_{i}>m$. Let $g_{i}: \Omega^{m}(V) \rightarrow \Omega^{n_{i}}(V)$ be a cocycle representing $\gamma_{i}$ if $\gamma_{i} \neq 0$. Let $g_{i}=0$ otherwise. Then $\sum f \circ g_{i}-\theta$ factors through a projective. That is, there exists $g^{\prime}: \Omega^{m}(V) \rightarrow P_{m}$ such that $f g^{\prime}=$ 
$\theta-\sum f g_{i}$. Then

$$
\theta=f \circ\left(g^{\prime}+\sum g_{i}\right)
$$

as claimed. Notice that only in unusual cases such as $G$ cyclic or $G \cong(\mathbb{Z} / 2)^{2}$ and $p=2, M=K$, does $\mathscr{C}$ satisfy the condition of Proposition 3.1.

Example 3.5. Let $V$ be an indecomposable nonprojective $K G$-module. Let $\mathscr{C}$ be the full subcategory generated by the projective modules and the modules $\Omega^{n}(V)$ for all $n \in \mathbb{Z}$. That is, here we allow both negative and positive translates of $V$. In general, $\mathscr{C}$ will not be contravariantly finite. For example, suppose that $V=K$, the trivial $K G$-module. Then the question involves the finite generation of negative cohomology, and often cup products in negative degrees are zero. More specifically, assume that $p=2$ and $G=\langle x, y\rangle$ is elementary abelian of order 4 . Let $M=K G(x-1)$. It can be checked that for any integer $m<0$ there is a surjective homomorphism $\gamma_{m}: \Omega^{m}(K) \rightarrow M$. On the other hand, if $n<m$ then any homomorphism $\phi: \Omega^{n}(K) \rightarrow \Omega^{m}(K)$ has $\phi\left(\Omega^{n}(K)\right) \subseteq \operatorname{Rad} \Omega^{m}(K)$. So suppose that we had an approximation $f: X \rightarrow M$ where $X=\amalg X_{i}, X_{i} \cong \Omega^{n_{i}}(K)$ for some $n_{i}$. Choose $m$ so that $m<n_{i}$ for all $i$. Then $\gamma_{m}$ cannot factor through $f$. A similar method can be used for other groups. However in the particular case of the group of order 4 , the result is also implied by the fact that the collection of objects in $\mathscr{C}$ is an entire Auslander-Reiten component.

\section{REFERENCES}

1. M. Auslander, Applications of morphism determined by objects, Representation Theory of Algebras, Lecture Notes in Pure and Appl. Math., vol. 37, Marcel Dekker, New York, 1976, pp. 254-327.

2. M. Auslander and I. Reiten, Representations of Artin algebras. III, Comm. Algebra 3 (1975), 239-294.

3. __ Applications of homologically finite subcategories, Adv. in Math. 86 (1991), 111-152.

4. M. Auslander and S. O. Smalø, Preprojective modules over Artin algebras, J. Algebra 66 (1980), 61-122.

5. __ Almost split sequences in subcategories, J. Algebra 69 (1981), 426-454.

6. J. F. Carlson, Projective resolutions and degree shifting for cohomology and group rings, Proceedings of the Tsukuba Workshop on Representations of Algebras (to appear).

7. L. Evens, The cohomology ring of a finite group, Trans. Amer. Math. Soc. 101 (1961), 224-239.

8. M. Kleiner, On the almost split sequences of relatively projective modules over a finite group (to appear).

9. R. Knörr, Relative projective covers, Proc. Sympos. Modular Representations of Finite Groups, Aarhus Univ., 1978, pp. 28-32.

Department of Mathematics, University of Georgia, Athens, Georgia 30602

E-mail address: jfc@joe.math.uga.edu

FAKUltät Für Mathematik, Universität Bielefeld, D-4800 Bielefeld, Germany

E-mail address: happel@math1.mathematik.uni-bielefeld.de 